\title{
Editorial
}

Giuseppe Lippi and Mario Plebani

\section{A modern and pragmatic definition of Laboratory Medicine}

https://doi.org/10.1515/cclm-2020-0114

Herein we propose a modern and pragmatic definition of Laboratory Medicine. Laboratory Medicine may henceforth be defined as "a clinical science and discipline, devoted to the quantitative measurement, or qualitative assessment, of any substance which can be assayed in any type of biological fluid of any animal species, thus including humans, for either medical or research purposes. The results of these measurements are translated into actionable information for improving the care and/or maintaining the wellness of both a single individual and an entire population". This definition implicitly encompasses the use of a vast array of analytical methods, especially including - though not essentially limited to - colorimetric, enzymatic, turbidimetric, electrochemical, immunochemical, separation, nuclear magnetic resonance, cytochemical, clotting, molecular, cytogenetic and flow cytometry techniques [1]. These tests can be used for the screening, diagnosis, staging, prognostication and therapeutic monitoring of a kaleidoscope of pathological conditions, as well as for establishing health, fitness and predisposition towards certain activities and behaviors.

Whilst we favorably acknowledge that this advocated definition may stimulate additional and constructive debate on this matter, we also hope that it may be seen as a good starting point for widespread discussion and/ or endorsement. We further refer to the EFLM document
"Syllabus for postgraduate education and training for Specialists in Laboratory Medicine" for garnering specific indications on the skills, knowledge and competence of specialists in Laboratory Medicine [1].

Author contributions: All the authors have accepted responsibility for the entire content of this submitted manuscript and approved submission.

Research funding: None declared.

Employment or leadership: None declared.

Honorarium: None declared.

\section{Reference}

1. Jassam N, Lake J, Dabrowska M, Queralto J, Rizos D, Lichtinghagen R, et al. The European Federation of Clinical Chemistry and Laboratory Medicine syllabus for postgraduate education and training for Specialists in Laboratory Medicine: version 5 - 2018. Clin Chem Lab Med 2018;56:1846-63.

Corresponding author: Prof. Giuseppe Lippi, Associate Editor of Diagnosis, Section of Clinical Biochemistry, Department of Neuroscience, Biomedicine and Movement, University Hospital of Verona, Piazzale L.A. Scuro, 10, 37134 Verona, Italy, Phone: 0039-045-8122970, Fax: 0039-045-8124308, E-mail: giuseppe.lippi@univr.it

Mario Plebani: Editor in Chief of Diagnosis, Department of Laboratory Medicine, University Hospital of Padova, Padova, Italy 